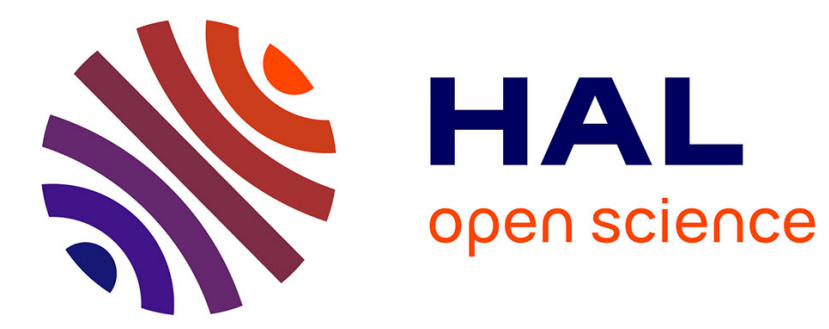

\title{
STRUCTURAL AND THERMODYNAMIC PROPERTIES OF LIQUID Zn, Ga, Sn FROM PAIR POTENTIALS
}

\author{
C. Regnaut, Jean Pierre Badiali, M. Dupont
}

\section{- To cite this version:}

C. Regnaut, Jean Pierre Badiali, M. Dupont. STRUCTURAL AND THERMODYNAMIC PROPERTIES OF LIQUID Zn, Ga, Sn FROM PAIR POTENTIALS. Journal de Physique Colloques, 1980, 41 (C8), pp.C8-603-C8-606. 10.1051/jphyscol:19808153 . jpa-00220253

HAL Id: jpa-00220253

https://hal.science/jpa-00220253

Submitted on 1 Jan 1980

HAL is a multi-disciplinary open access archive for the deposit and dissemination of scientific research documents, whether they are published or not. The documents may come from teaching and research institutions in France or abroad, or from public or private research centers.
L'archive ouverte pluridisciplinaire HAL, est destinée au dépôt et à la diffusion de documents scientifiques de niveau recherche, publiés ou non, émanant des établissements d'enseignement et de recherche français ou étrangers, des laboratoires publics ou privés. 
The interpretation of the "anomalous" structure factors of $\mathrm{Zn}$, Ga, $\mathrm{Sn}$ from long range pair potential agrees with liquid thermodynamic properties. Polymorphism and phases change of $\mathrm{Ga}$ are also explained with the same model.

Recent progress have been done in the analysis of the liquid metals structure factors $S(q)$, within the framework of the pseudopotential theory. Simulation and perturbation techniques have been used to calculate $S(q)$ from full metallic pair potentials, including the long range oscillating part

The hard sphere like profiles of the $\mathrm{Mg}$, $\mathrm{Al}$, and alkalis structure factors have been explained from ab ivitio pseudoputentials [1,2]. Shoulder or asymmetry in the main peak of the $\mathrm{Ga}, \mathrm{Sn}, \mathrm{Zn}$ structure factors have been interpreted on the same basis [2,3], or from ionic polarisability [4].

However, calculated $\mathrm{S}(\mathrm{q})$ are rather sensitive to the choice of the pseudopotential in these "anomalous" metals ( $\mathrm{Zn}, \mathrm{Ga}, \mathrm{Sn})$. Such a sensitivity to the pseudopotential also appears in other structural properties, for instance, in the determination of the crystal structure stability of Ga $[5,6,7]$. The usefullness of a pseudopotential approach really emerges if various properties of condensed matter can be described from the same model.

The purpose of this work is to compare results, from local and non local pseudopotentials, for several equilibrium properties : structure factor, 1iquid thermodynamic properties near the melting temperature, crystalline phases stabilities and high pressure phases transitions of gallium.

We briefly present the models of pair potentials and their corresponding theoretical $s(q)$.

The thermodynamic properties may be calculated either with the variational approach VA [8], or through the "optimized random phase approximation" ORPA [9]. The ORPA supersedes the VA for Lennard Jones fluids [10] but it has not been applied, to our knowledge, in liquid metals. Results from the two methods are compared here.

The analysis of the stable crystal structure for $\mathrm{Ga}$ is a sensitive test for any pseudopotential. We finally compare the zero temperature binding energies of four observed and one hypothetical (FCC) Ga structures.

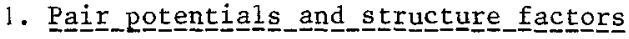

For each liquid metals, we consider three pair potentials : The ECM of Ashcroft [1]], the OMP of Shaw [12], and the renormalized form with effective masses, here labelled OMP 2 [13]. The exchange and correlation between electrons are included via the Vashishta-Singwi function [14]. At the liquid metal density, the pair potentials obtained have comnon features : Fxiedel oscillations at long range, and at short range a first minimum at a distance $r_{0}$ close to the first peak position of the liquid radial distribution function. The first minimum amplitude $V\left(r_{0}\right)$ and $r_{0}$ depends on the models (Table 1).

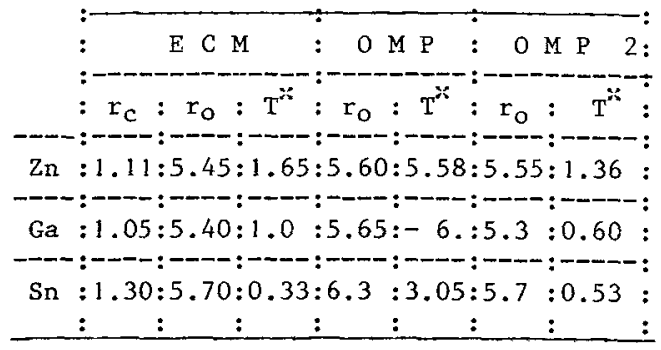

Table 1 : First minimum position $r_{0}$ and reduced temperature $\mathrm{T}^{2}=\mathrm{k}_{\mathrm{B}} \mathrm{T} / \mathrm{V}\left(\mathrm{r}_{\mathrm{O}}\right)$ for the three models. The ECM radius $r_{c}$ and $r_{0}$ are in atomic unit.

The structure factors have been derived by the ORPA technique. The pair potentials are truncated at a mode close to $4 \mathrm{r}_{\mathrm{o}}$. The numerical procedure and comparison to Monte Carlo method for metal1ic potentials have been discussed elsewhere [15]. Quantitative agreement between ORPA and Monte Carlo approaches is expected for reduced temperature higher than 0.5 (table 1). Excepted for the ECM Sn case, $T^{*}$ is always above this value. 
In the figure 1, we compare the Sn calculated structure factors to experiment [16]. The others $\mathrm{S}(\mathrm{q})$ have been reported by $[2,3]$.

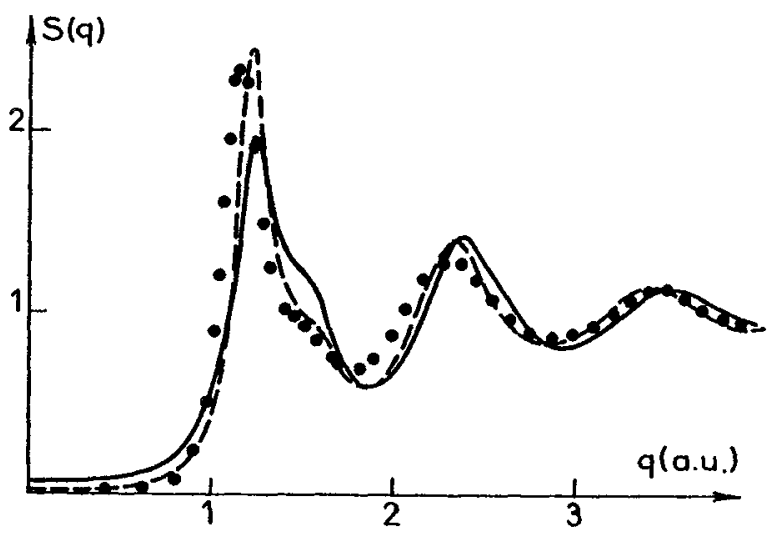

Fig.1 : Sn Structure factors

... Experiment model OMP 2

--- model OMP

For $\mathrm{Sn}$, the OMP leads to the best overall agreement with experiment. The ECM $S(q)$, not reported in the figure, exhibits very strong oscillations, and deviates from experiment. Such oscillations are related to the large oscillations of the pair potential, but in view of the low $\mathrm{T}^{2}$ value with the ECM, the ORPA procedure may be also suspicious.

For $\mathrm{Zn}$ we find that the OMP 2 better reproduces the asymmetry of the main peak in $\mathrm{S}(q)$. For $\mathrm{Ga}$ this model gives qualitatively the expected shoulder in $S(q)$. This suggests that effective masses are important for the structural behaviour of this two metals.

2. Liquid_thermodynamic_properties_es_near mel melting point

The pseudopotential calculation of liquid metals thermodynamic properties are currently done with a variational determination of the free energy of a hard sphere reference system [17]. However, the ORPA calculation of $S(q)$ may be self consistently done with the optimization of the free energy of a reference system which includes the long range part of the pair potential [9].

Calculated thermodynamic properties of the Lennard Jones liquid, from the ORPA, are in much better agreement with computer experiments, than those of the variational approach [10]. Thus, in this work, we develop our calculations within the ORPA.

For metallic systems, the total ion free energy may be expressed in q-space, and in the ORPA scheme we derive the following expression in atomic units :

$$
\begin{aligned}
F= & Z^{x}\left(\frac{3}{5} E_{F}+E_{x c}+\frac{A_{c}}{\Omega}\right)+F_{c e}+F_{g}+F_{\sigma}+U_{M}(\sigma) \\
(1) & +U_{B S}(\sigma)+\frac{2 \pi}{\Omega} \int_{\sigma}^{r_{0}} r^{2}\left[V\left(r_{0}\right)-V(r)\right] g_{\sigma}(r) d r \\
& +\frac{\Omega k_{B} T}{4 \pi^{2}} \int_{0}^{\infty}\left[1-\frac{S_{\sigma}(q)}{S_{t}(q)}+\log \frac{S_{\sigma}(q)}{S_{t}(q)}\right] q^{2} d q
\end{aligned}
$$

In (1), the terms in brackets are zero and first order terms in the perturbation energy expansion, $F_{e e}$ is the electronic free energy, $F_{g}$ the perfect gas contribution. All these terms are independent of the liquid structure. The hard sphere free energy $F_{\sigma}$, the Madelung energy $U_{M}(\sigma)$ and the band structure energy $\mathrm{J}_{\mathrm{BS}}(\sigma)$ are defined in [17], but here the hard sphere diameter $\sigma$ is determined from the ORPA procedure. The last two corrective terms in (1) depend on the hard sphere radial distribution function $g_{\sigma}(r)$ and on reference $s_{t}(q)$ and hard sphere $S_{\sigma}(q)$ structure factors.

When the ECM is used, the effective valence $Z:$ is the real valence. As noted in [i]], the core term $A_{c}$ must be adjusted in order to ensure normal (i.e. nearly zero) pressure at the observed liquid volume $\Omega$. Such uncertainty also remains with OMP and OMP 2 [18], and we come back to simplification by considering $A_{C}$ as an independent parameter in these models.

In the VA expression of the free energy [17], there is no corrective terms and $\sigma$ is determined in another way. At first, it is interesting to investigate the ORPA and VA differences for liquid metals. We have done this for the ECM pseudopotential. We calculate the VA and ORPA free energies and their partial derivatives as functions of the volume and the temperature. Of course, the adjustment of the $A_{c}$ term is on1y done in one of the two determination of the free energy at zero pressure (here for the VA).

The table 2 summarizes the results with the ECM.

The rather unreliable results for $\mathrm{Sn}$ again reflect a possible failure of the ORPA procedure or ECM pair potential. For $\mathrm{Zn}$ and $\mathrm{Ga}$, we find that the VA values deviate from the ORPA values as in Lennard Jones fluids of the same density and reduced temperature [10]. 


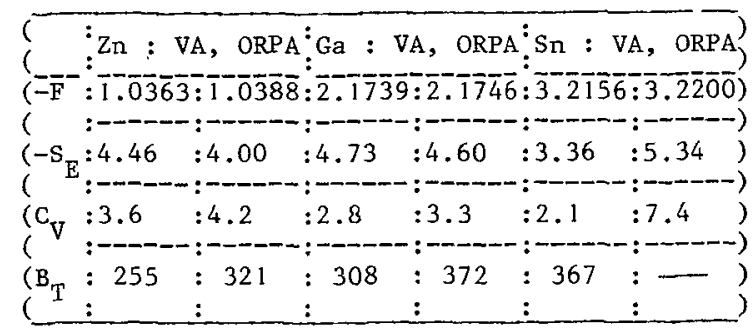

Table 2 : Comparison between variational approach and ORPA results for liquid $\mathrm{Zn}(723 \mathrm{~K})$, $\mathrm{Ga}$ (293K), Sn (523 K).

Free energy is in atomic unit (e $=\nVdash=m=1)$, entropy $\mathrm{S}_{\mathrm{E}}$ and specific heat $\mathrm{C}_{V}$ are in $\mathrm{k}_{\mathrm{B}}$ unit. Bulk modulus is in $\mathrm{Kbar}$.

The thermodynanical results within the ORPA scheme are presented in table 3 , in order to compare the ECM, OMP and OMP 2.

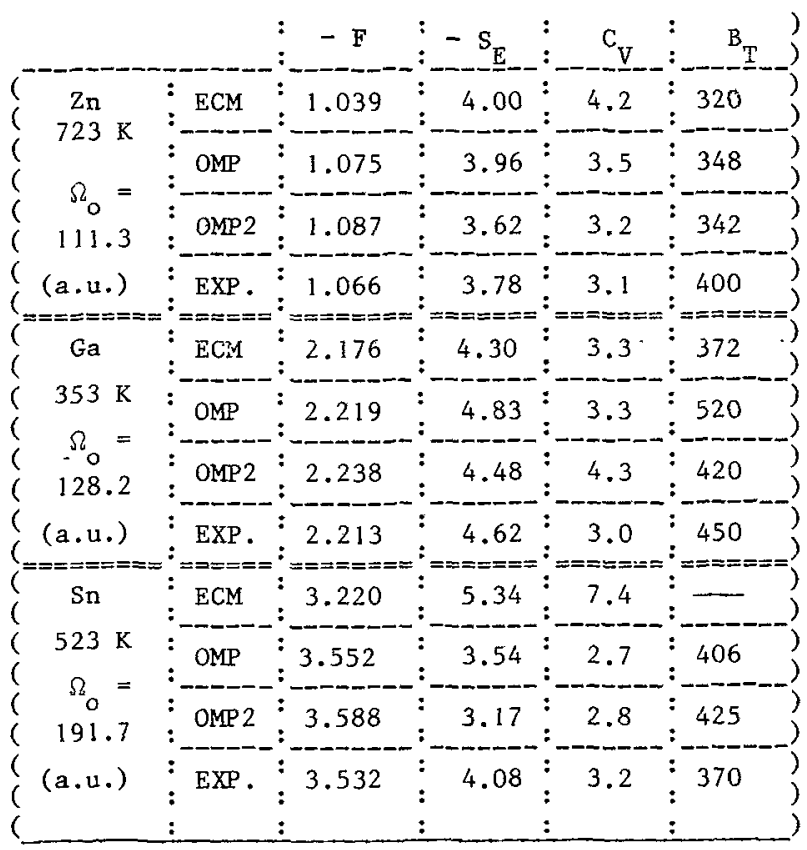

Table 3 : ORPA results. The core term $A_{c}$ is adjusted for each model to assume zero pressure at the observed volume $\Omega_{0}$. Free energies experimental values are determined using the experimental cohesive energy values of [19] and the harmonic Debye approximation for the vibrational free energy of solid at melting. Experimental values of $S_{E}, C_{V}$ and $B_{T}$ are respectively from $[20],[21]$ and $[22,23]$. Units are as in table 2 .

Clearly, for $\mathrm{Sn}$ and $\mathrm{Zn}$, the OMP or the OMP2 give results which are quantitatively closer to experiment than the ECM ones. We observe that the best agreement for entropy is obtained for the models which also reproduce the anomalies of the structure factors of $\mathrm{Zn}, \mathrm{Ga}, \mathrm{Sn}$.

Calculations have been done in litterature with the WCA approximation [21]. We notice by comparison that including the long range forces via ORPA leads to a non negligible difference between WCA and ORPA results.

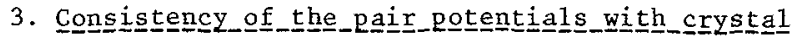

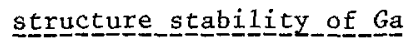

Comparison of the different crystal structure energies has been prooved to be a test for the pseudopotential approach for $\mathrm{Ga}[5,6,7]$.

Here we consider the two phases $\alpha-G a\left(\Omega_{0}=\right.$ 131 a.u.) and $B-G a\left(\Omega_{o}=124\right.$ a.u. $)$ which are observed under normal pressure, and two high pressure structures II-Ga $\left(\Omega_{0}=118\right.$ a.u. $)$ and III-Ga $\left(\Omega_{0}=118\right.$ a.u. $)$ which have been recently measured [24]. Moreover, we also consider a FCC structure predicted but not yet observed at very high pressure [25]. The $\alpha-G a$ stable structure has the lowest energy at zero pressure. The extrapolation of the equilibrium phases diagramm at OK $[5,6,7]$ shows that three high pressure phase transitions may occur : near $30 \mathrm{Kbar} \alpha-\mathrm{Ga} \rightarrow \mathrm{II}-\mathrm{Ga}$ and $\alpha-\mathrm{Ga} \rightarrow \beta-\mathrm{Ga}$, and near $100 \mathrm{Kbar} \beta-\mathrm{Ga} \rightarrow \mathrm{III}-\mathrm{Ga}$.

In a zero temperature analysis, the internal energy of solid is the sum of the first three terms in brackets in (1) plus the Madelung $U_{M}$ and the band structure $U_{B S}$ energies. The numerical determination of $U_{M}$ and $U_{B S}$ follows the standard methods $[6,7]$ and are done for any of the three mode1s ECM, OMP, OMP2, either with the Vashista-Singwi exchange correlation function or with the ToigoWodruff one [26]. The zero point vibrational energy is neglected. The internal energy versus volume curves are presented in fig. 2 and correspond to the best results obtained among the three pseudopotentials and the two exchange correlation functions.

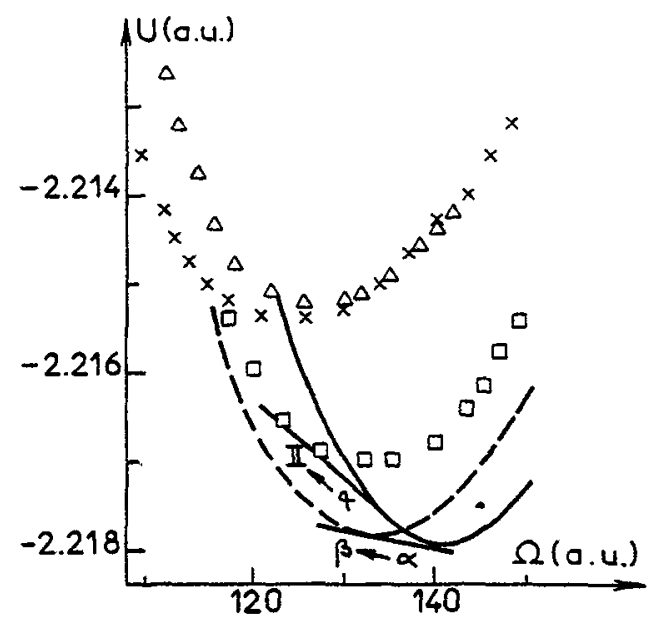

Fig. 2 : Internal energy ( $T=0 K$ ) vs volume for Ga : $-\alpha,--\beta, \square D I I, \Delta \Delta$ III, xx hypothetical FCC. 
The ECM neither predicts the $\alpha-G a$ structure to be the most stable, nor the correct phase transitions. A contrario, the OMP2 combined with the ToigoWoodruff function (This gives also a slight better agreement between experiment and theory for $S(q)$ leads to the general trends of the energy phase diagram. More precisely $\alpha-G a$ is the most stable one, the calculated equilibrium volume being however $7 \%$ too high; the II and III forms are correctly unstable at zero pressure; the phase transitions $\alpha \mathrm{Ga} \rightarrow \beta \mathrm{Ga}$ and $\alpha \mathrm{Ga} \rightarrow$ II Ga are predicted from the common tangent construction, but only the second one occurs near the expected pressure of $30 \mathrm{Kbar}$; the $\beta \mathrm{Ga} \rightarrow \mathrm{III}$ Ga pressure transition is found to be near $110 \mathrm{Kbar}$.

Present results for solid Ga, obtained with no empirical adjustment of the pseusopotential in the structural energy calculations are as successfull as other calculations [5-7].

\section{Conclusion}

"Anomalous" properties of liquid metals $\mathrm{Zn}$, Ga, Sn have been investigated from the pseudo-potential theory. We emphasize that the peculiarities such as shoulder or asymetry in structure factor, and the thermodynamic properties may be explained assuming a long range pair potential. The ORPA method has been used to compare the results for three models of pair potentials. We find that the non local Shaw's models better predicts the expected features than Aschroft's mode1. Study of gallium polymorphism supports the present analysis.

\section{References}

[1] R.S. DAY, F. SUN, and P.H. CUTLER Phys. Rev. A, 19 (1979), 328.

[2] C. REGNAUT, J.P. BADIALI and M. DUPONT Phys. Lett. 74A, 3,4 (1979) 245 and errata 75A, 6 (1980), 516.

[3] R. OBERLE and H. BECK Sol. Stat. Comm. 32, (1979), 959.

[4] K.K. MON, N.W. ASHCROFT and G.V. CHESTER Phys. Rev. B 19 (1979), 5103.

[5] J. HAFNER and O. HITMAIR, Osterr. Akad. Wiss. Math. Naturwis's. 4, (1975), 497.

[6] J.E. INGLESFIELD, J. Phys. C. Sol. Stat. Phys. 1, (1968), 1337 .

[7] M. DUPONT Thesis Paris (1976).

[8] G.A. MANSOORI and F.B. CANEIELD J. Chem. Phys, 51, (1969), 11.
[9] H.CANDERSEN, D. CHANDLER and J.D. WEEKS J. Chem. Phys. 56, 8 (1972) 3812.

[10] J.A. BARKER, D. HENDERSON Rev. Mod. Phys. 48,4, (1976), 587.

[11] N.W. ASHCROFT, Phys. Lett. 23 (1966), 48.

[12] R.W. SHAW Jr., J. Phys. C. Sol. St. Phys. 2, 2, (1969), 2335.

[13] R.W. SHAW Jr. J. Phys. C. Sol. St. Phys. 2, 2 (1969), 2350.

[14] P. VASHISHTA and K.S. SINGWI Phys. Rev. B6, (1972), 875.

[15] C. REGNAUT, M. DUPONT and J.P. BADIALI Poster Session in "RCP Liquides et amorphes métalliques" Grenoble (1979).

[16] Y. WASEDA Private comm.

[17] N.W. ASHCROFT and D. STROUD So1. Stat. Phys. 33, (1978), 1.

[18] R. PYNN, Phys. Rev. B 5, 12 (1972), 4826.

[19] J. HAFNER, Phys, Stat. Sol. (b), 57 (1973), 101

[20] R. HULTGREN, R.L. ORR, P.D. ANDERSON and K.K. KELLY in Selected Values of Thermodynamic properties of metals and alloys" New York WILEY (1963).

[21] R. KUMARAVADIVEL and R. EVANS J. Phys. C., 9, (1976), 3877.

[22] T.E. FABER in "Introduction in the theory of liquid metals" Cambridge Univ. Press (1972)

[23] H. KÖSTER, F. HENSEL, E.U. FRANCK Ber. Buns. Ges. 74, 1, (1970), 43.

[24] L. BOSIO, J. Chem. Phys. 68, 3, (1978), 1221.

[25] B. PREDEL, J. Chim. Phys. 9, (1977), 959.

[26] F. TOIGO and T.O. WOODRUFF Phys. Rev. B2, (1970) 3958. 